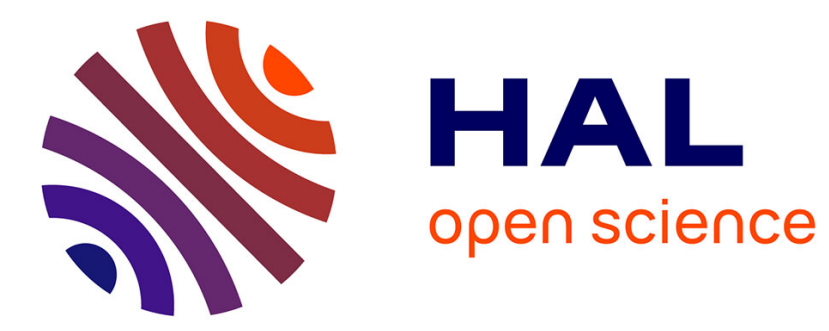

\title{
Influence de la préoxydation d'un PEHD sur l'extraction des carbonyles et la croissance d'un biofilm
}

C Pons, F Farcas, Emmanuel Richaud, Bruno Fayolle, T Bouchez, L Mazeas

\section{To cite this version:}

C Pons, F Farcas, Emmanuel Richaud, Bruno Fayolle, T Bouchez, et al.. Influence de la préoxydation d'un PEHD sur l'extraction des carbonyles et la croissance d'un biofilm. Matériaux \& Techniques, 2012, 100, pp.211-220. 10.1051/mattech/2012018 . hal-01202711

\section{HAL Id: hal-01202711 \\ https://hal.science/hal-01202711}

Submitted on 21 Sep 2015

HAL is a multi-disciplinary open access archive for the deposit and dissemination of scientific research documents, whether they are published or not. The documents may come from teaching and research institutions in France or abroad, or from public or private research centers.
L'archive ouverte pluridisciplinaire HAL, est destinée au dépôt et à la diffusion de documents scientifiques de niveau recherche, publiés ou non, émanant des établissements d'enseignement et de recherche français ou étrangers, des laboratoires publics ou privés. 


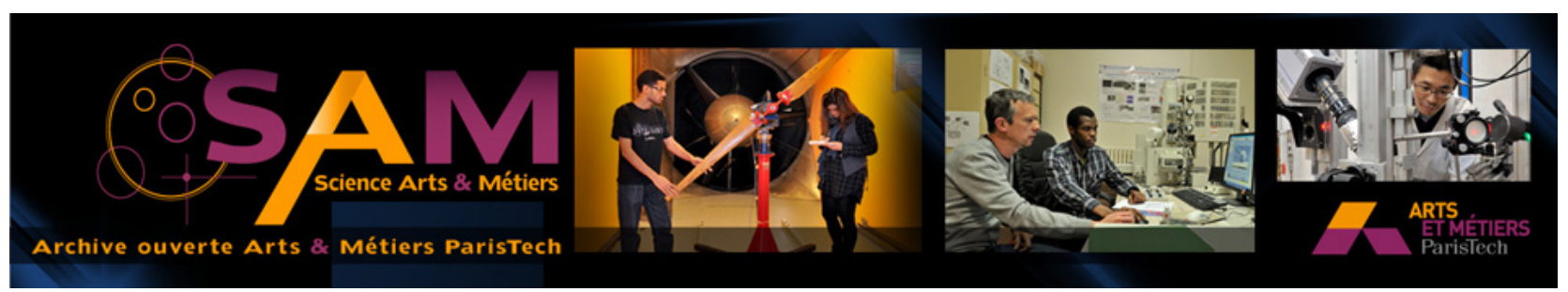

\section{Science Arts \& Métiers (SAM)}

is an open access repository that collects the work of Arts et Métiers ParisTech researchers and makes it freely available over the web where possible.

This is an author-deposited version published in: http://sam.ensam.eu

Handle ID: .http://hdl.handle.net/10985/10085

\section{To cite this version :}

C PONS, F FARCAS, Emmanuel RICHAUD, Bruno FAYOLLE, T BOUCHEZ, L MAZEAS Influence de la préoxydation d'un PEHD sur l'extraction des carbonyles et la croissance d'un biofilm - Materiaux Et Techniques - Vol. 100, p.211-220. - 2012 


\title{
Influence de la préoxydation d'un PEHD sur l'extraction des carbonyles et la croissance d'un biofilm
}

\author{
C. Pons ${ }^{1}$, F. Farcas ${ }^{1}$, E. Richaud ${ }^{2}$, B. Fayolle ${ }^{2}$, T. Bouchez ${ }^{3}$ et L. Mazeas ${ }^{3}$ \\ 1 Ifsttar, Département Matériaux, Groupe Comportement Physico-chimique et Durabilité des \\ Matériaux, 58 Bd Lefebvre, 75732 Paris Cedex 15, France \\ e-mail : carlota.pons@ifsttar.fr; fabienne.farcas@ifsttar.fr \\ 2 PIMM (UMR CNRS 8006), Arts et Métiers ParisTech, 151 Bd de l'hôpital, 75013 Paris, France \\ 3 Cemagref, unité de recherche Hydrosystèmes et bioprocédés, Parc de Tourvoie, BP 44, \\ 92163 Antony, France
}

Mots-clés :

PEHD; biofilm ; carbonyles ; IRTF ; MEB

Key words:

HDPE; biofilm; carbonyls; FTIR; SEM

\begin{abstract}
Résumé - Les géomembranes en polyéthylène haute densité (PEHD) sont utilisées comme barrière d'étanchéité dans les Installations de Stockage de Déchets Non Dangereux (ISDND). Malgré les bonnes propriétés initiales du PEHD, face aux agressions chimiques et biologiques du lixiviat, sa durabilité reste une question ouverte. L'objectif de cette étude est de déterminer la nature des espèces carbonylées bioassimilables qui ont été extraites d'un PEHD préoxydé et incubé 7 mois à $40^{\circ} \mathrm{C}$ dans un lixiviat d'ISDND. L'identification des produits d'oxydation du PEHD, éventuels substrats pour le développement de microorganismes, est comparée à la croissance du biofilm formé à la surface du polymère. La nature et la quantité des espèces carbonylées extraites du polymère sont déterminées par spectrophotométrie infra-rouge à transformée de Fourier (IRTF) en mode transmission, avant et après dérivatisation chimique. Parallèlement, la croissance du biofilm est suivie par IRTF en mode réflexion totale atténuée (ATR) ainsi que par Microscopie Électronique à Balayage (MEB).
\end{abstract}

\begin{abstract}
Extraction of oxidation compounds and biofilm growth of pre oxidized polyethelene. High-density polyethylene (HDPE) geomembranes are used as a sealing barrier in the municipal solid waste (MSW) landfills. Despite good initial properties of the HDPE, its durability limited by chemical and biological ageing in MSW landfill leachate, remains an open question. The aim of this study is to determine the nature of the carbonyl compounds generated by the preodixation process and extracted from an HDPE film preoxidized and incubated 7 months at $40{ }^{\circ} \mathrm{C}$ in a municipal solid waste (MSW) landfill leachate. The identification of the HDPE oxidation products as carboxylic acids, potential substrates for the development of microorganisms, and its depletion kinetic are compared to the biofilm growth formed on the polymer surface. The nature and concentration of extracts carbonyl compounds are determined by Fourier Transformed Infra-Red (FTIR) spectroscopy in transmittance mode, before and after chemical derivatization. In parallel, the biofilm growth at the HDPE surface is followed by attenuated total reflection FTIR spectroscopy and by Scanning Electron Microscopy (SEM).
\end{abstract}

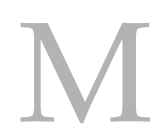
algré les efforts de développement des filières de recyclage et de réutilisation, le stockage constitue encore aujourd'hui dans la plupart des pays, le mode d'élimination majoritaire des déchets non dangereux. Afin de diminuer les risques environnementaux, les nouvelles dispositions réglementaires (Arrêté Ministériel du 9 septembre 1997, mis à jour du 19 janvier 2006), imposent la mise en place d'un système d'étanchéité en fond et flancs d'alvéoles d'installation de stockage de déchets (ISDND). Ces systèmes sont notam- ment composés d'une géomembrane généralement en polyéthylène haute densité (PEHD) du fait de sa grande inertie chimique et biologique.

Il est important de préciser que bien que l'ASQUAL $^{1}$ et le Comité Français des Géosynthétiques (CFG) utilisent la dénomination "géomembranes en PEHD », en

1 ASQUAL : Association qualité sans but lucratif, créée par des Centres Techniques auxquels se sont joints le Comité Français des Géosynthétiques (CFG) et le Comité Français des Agrotextiles. 
Europe ces géomembranes utilisées dans les ISDND sont en général composées de différents types de résines à bases de polyéthylène (PEHD ou PEBD ou PEBDL), auxquelles sont ajoutées des additifs. Le terme PEHD utilisé ne doit donc pas être assimilé à des polymères de PEHD dont les propriétés chimiques et mécaniques sont différentes de celles des polymères de PE utilisés dans les géomembranes situées dans les ISDND.

En fond d'installation de stockage de déchets, les géomembranes en PEHD ne sont pas soumises à des phénomènes de photooxydation mais subissent des contraintes thermiques (entre $30{ }^{\circ} \mathrm{C}$ et $40^{\circ} \mathrm{C}$ ) $[1,2]$ générées par les réactions de dégradation des déchets, et sont en permanence au contact d'une population riche et diversifiée de microorganismes. Des températures plus élevées (de l'ordre de $50{ }^{\circ} \mathrm{C}$ à $60{ }^{\circ} \mathrm{C}$ ) ont été observées sur des sites ne possédant pas de système de récupération des lixiviats ou sur des sites où des ajouts de lixiviats ont été effectués afin d'accélérer la biodégradation des déchets $[3,4]$.

Bien que les polyéthylènes soient considérés comme chimiquement et biologiquement inertes, de nombreuses études montrent qu'une fois oxydés, ils peuvent être attaqués par des microorganismes [5-12] et qu'il existe un effet synergique entre leur état d'oxydation et leur biodégradation [13]. En effet, les mécanismes d'oxydation génèrent des composés carbonylés qui résultent de ruptures de chaînes moléculaires des polymères naturels et synthétiques.

La thermo oxydation des PE est un processus radicalaire en chaîne auto catalytique $[14,15]$ gouverné par la concentration en oxygène ou la température [16]. La réaction est amorcée par un paramètre extrinsèque, tel qu'une impureté au sein du matériau, qui induit la formation de radicaux libres ou d'hydroperoxydes. Ces derniers ont un rôle clé puisqu'ils apparaissent comme le premier produit qui, par sa décomposition entraine la formation de la plupart des composés carbonylés (acides carboxyliques, cétones, esters... ) [7]. Les composés carbonylés de plus faibles tailles moléculaires que le polymère d'origine sont bioassimilables. L'étape d'oxydation semble donc conditionner la biodégradabilité des polymères.

L'utilisation du polymère comme source de carbone par les microorganismes est facilitée par la formation d'un biofilm à la surface du polymère [18]. Initialement, la nature hydrophobe du PE ne permet pas la fixation des microorganismes à sa surface. Après un vieillissement oxydatif, le caractère hydrophile de la surface du PE augmente et un biofilm peut alors se former.

La majorité des études sur la biodégradation du PE ont été effectuées dans des milieux contenant des souches bactériennes pures $[6,7,9]$ ou des composts [10, 12, 19], mais aucune n'a encore été effectuée dans un milieu aussi complexe que le lixiviat d'ISDND.

Afin de connaître les mécanismes de vieillissement des PE dans les ISDND, l'objectif de cette étude est de déterminer la nature des espèces carbonylées bio assimilables extraites d'un PEHD préoxydé et incubé 7 mois à $40{ }^{\circ} \mathrm{C}$ dans un lixiviat d'ISDND. L'identification des produits d'oxydation est comparée à la croissance d'un biofilm à la surface du polymère.

\section{Matériaux et méthodes}

\subsection{Matériaux de l'étude}

Le polyéthylène haute densité (PEHD) utilisé dans cette étude est un polymère sans additif. Il présente les caractéristiques suivantes : $M_{w}=214512 \mathrm{~g} \cdot \mathrm{mol}^{-1} ; M_{n}=$ 13261 g.mol ${ }^{-1} ; P I=16,2$. L'étude a été réalisée sur des films d'une épaisseur d'environ $300 \mu \mathrm{m}(+/-30 \mu \mathrm{m})$, obtenus par pressage à chaud à $200{ }^{\circ} \mathrm{C}$ sous une pression de 30 bars durant 90 s. Puis les films ont été conservés à basse température $\left(+4^{\circ} \mathrm{C}\right)$ avant vieillissement.

\subsection{Préoxydation des films de polyéthylène}

Bien que la quantité d'oxygène et l'état d'oxydation des géomembranes dans le fond des alvéoles d'ISDND soient faibles, nous avons choisi de nous placer à un niveau d'oxydation suffisamment élevé pour nous permettre de mettre en évidence l'évolution 


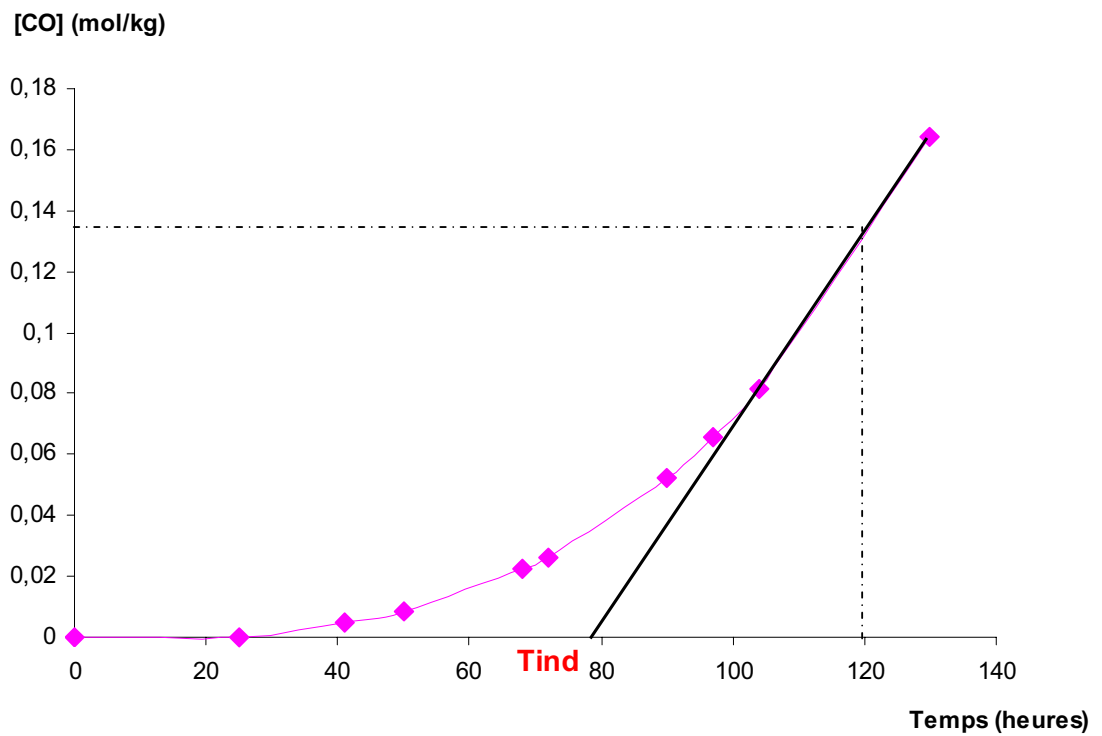

Fig. 1. Variation de la concentration en carbonyles en fonction du temps à $105^{\circ} \mathrm{C}$.

Fig. 1. Variation of carbonyl concentration with time at $105^{\circ} \mathrm{C}$.

de la concentration des fonctions carbonyles du PEHD mis au contact d'un lixiviat.

En se basant sur les conditions préconisées dans la norme NF EN ISO 13438 des géosynthétiques, l'oxydation du PEHD a été accélérée en laboratoire à une température de $105^{\circ} \mathrm{C}$ dans une étuve sous air à la pression atmosphérique. Le niveau d'oxydation a été déterminé en suivant l'augmentation de la concentration en fonctions carbonyles par infra-rouge à transformée de Fourier (IRTF), au cours du temps (Fig. 1). Pour déterminer leur concentration, nous avons appliqué la loi de Beer-Lambert (Éq. (1)) au pic maximum situé à $1715 \mathrm{~cm}^{-1}$ :

$$
[\mathrm{CO}]=\frac{\mathrm{DO}_{1715}}{\varepsilon_{1715} \mathrm{dl}}
$$

où :

- [CO] représente la concentration en groupements carbonyles (en mol. $\mathrm{kg}^{-1}$ ) ;

- l est l'épaisseur de l'échantillon (en cm);

- $d$ est la densité du PE, prise égale à $0,95 \mathrm{~kg} .1^{-1}$;

- $\varepsilon_{1715}$ est le coefficient d'extinction molaire : $\varepsilon_{1715}=3001 \cdot \mathrm{mol}^{-1} \cdot \mathrm{cm}^{-1}$ [20].

Nous nous sommes placés à une concentration en carbonyles [CO] $=1,3 \times 10^{-1}$ mol. $\mathrm{kg}^{-1}$ c'est-à-dire après la période d'induction à l'oxydation $T_{\text {ind }}$ (Fig. 1). On considérera donc nos échantillons comme préoxydés.

\subsection{Milieux et conditions de vieillissement}

\subsubsection{Milieux de vieillissement}

Les échantillons de PEHD ont été immergés dans un lixiviat de déchets âgés de 10 ans. Le lixiviat a été récupéré au fond d'une alvéole à l'aide d'un système de drainage étanche présent en fond de site. Le lixiviat récupéré présente un $\mathrm{pH}$ de 8 .

Afin de mettre en évidence l'action des bactéries d'un lixiviat de déchets sur la dégradation de films de PEHD préoxydés, les vieillissements ont été réalisés par incubation dans le lixiviat contenant des microorganismes (biotique) et dans le lixiviat exempt de microorganismes (abiotique).

Les milieux d'incubations abiotiques ont été obtenus par ajout d'un inhibiteur de croissance des microorganismes : le Thiomersal, en concentration finale $0,01 \%$ $(\mathrm{m} / \mathrm{m})$ [11].

\subsubsection{Conditions de vieillissement}

Les films de PEHD sont placés dans des flacons en verre de $30 \mathrm{ml}$ contenant $10 \mathrm{ml}$ de milieu d'incubation.

Les essais sont ensuite placés en étuve à $40{ }^{\circ} \mathrm{C}$ afin de se placer dans les conditions de 
températures de fond d'ISDND. Des échantillons de PEHD non préoxydés ont été utilisés comme référence dans chaque condition. Dans un souci de répétabilité, deux films sont placés dans chaque type de conditions.

\subsection{Méthodes d'analyse}

\subsubsection{Spectrophotométrie infra-rouge à transformée de Fourier mode transmission}

La spectrophotométrie infra-rouge à transformée de Fourier (FTIR) a été utilisée pour déterminer la formation ou la disparition de groupes fonctionnels au sein du polymère.

Les spectres infra-rouges ont été réalisés grâce à un spectromètre Nicolet Impact 400, piloté par le logiciel d'acquisition Omnic 5.1. Les spectres sont enregistrés avec une résolution de $4 \mathrm{~cm}^{-1}$ et une accumulation de 32 spectres.

L'oxydation en étuve et le vieillissement dans les lixiviats ont été suivis par l'évolution du massif des groupements carbonyles. L'absorbance des carbonyles est convertie en concentration par l'utilisation de loi de BeerLambert au pic maximum $\left(1715 \mathrm{~cm}^{-1}\right)$.

\subsubsection{Spectrophotométrie infra-rouge à transformée de Fourier mode ATR}

La spectrophotométrie IRTF-ATR a été utilisée pour suivre et analyser le développement des microorganismes via la croissance d'un biofilm à la surface des films de PEHD. Une attention particulière a été portée sur l'absorption des régions : 3000$3600 \mathrm{~cm}^{-1}$ et $1653 \mathrm{~cm}^{-1}$ dont les bandes correspondent au matériel protéique synthétisé par les microorganismes; ainsi que la région $1200-900 \mathrm{~cm}^{-1}$ attribuable aux polysaccharides, constituants cellulaires des microorganismes $[9,11,21]$.

\subsubsection{Microscopie électronique à balayage}

La croissance des microorganismes à la surface du polymère a été observée par Microscopie Électronique à Balayage (MEB), à l'aide d'un MEB de type Quanta 400 de chez FEI. Les films de PEHD ont été observés après métallisation à l'or sous vide grâce à un métalliseur Bal-Tec SCD004 Sputter Coater, sous plusieurs grossissements.

\section{Résultats et discussion}

\section{1 Évolution de la quantité de produits carbonylés d'un polyéthylène oxydé, vieilli dans un lixiviat d'ISDND}

Des échantillons de PEHD préoxydés puis incubés dans un lixiviat d'ISDND biotique ou abiotique, ont été analysés à différentes échéances par IRTF en mode transmission afin de suivre la quantité en produits carbonylés présente au sein du polymère.

L'incubation des films de PEHD préoxydés et incubés dans un lixiviat d'ISDND biotique et un lixiviat abiotique en conditions aérobies cause la disparition de la bande caractéristique des composés carbonylés du PEHD, située entre 1800 et $1650 \mathrm{~cm}^{-1}$ sur les spectres IRTF (Fig. 2). Cette diminution semble majoritairement due à une extraction physique de ces composés.

Pour modéliser et quantifier ce phénomène d'extraction, on peut en première approche utiliser la relation suivante [22] :

$$
\frac{\partial[\mathrm{CO}]}{\partial t}=-H[\mathrm{CO}]
$$

où :

- CO est la concentration en carbonyles résiduels (mol. $\left.\mathrm{L}^{-1}\right)$;

- $t$ est le temps (jours);

- $H$ est le coefficient d'extraction (jours ${ }^{-1}$ ).

La linéarité des courbes d'évolution du logarithme de la concentration en carbonyles du PE en fonction du temps, pour des échantillons de PEHD incubés dans le lixiviat d'ISDND et dans un lixiviat abiotique (Fig. 3) montre que la perte des carbonyles suit une cinétique du premier ordre. Le calcul des coefficients d'extraction, $H_{\text {Lixiviat biotique }} \sim 1,9 \times 10^{-2}$ jours $^{-1}$ pour le lixiviat biotique et $H_{\text {Lixiviat abiotique }}$ $1,3 \times 10^{-2}$ jours $^{-1}$ pour le lixiviat abiotique, montre que quelque soit le milieu considéré, les vitesses d'extraction sont comparables. Ces résultats sont en accord avec ceux obtenus par Koutny et al. [11] qui mettent en évidence l'extraction des composés carbonylés d'échantillons de PEHD et PEBD dans un milieu aqueux, par spectroscopie RMN, sans toutefois quantifier les vitesses d'extraction et les quantités de composés extraits. 


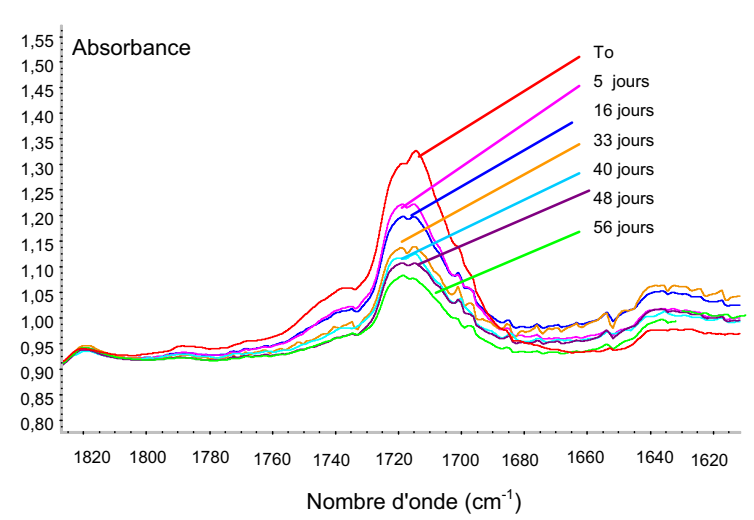

(a)

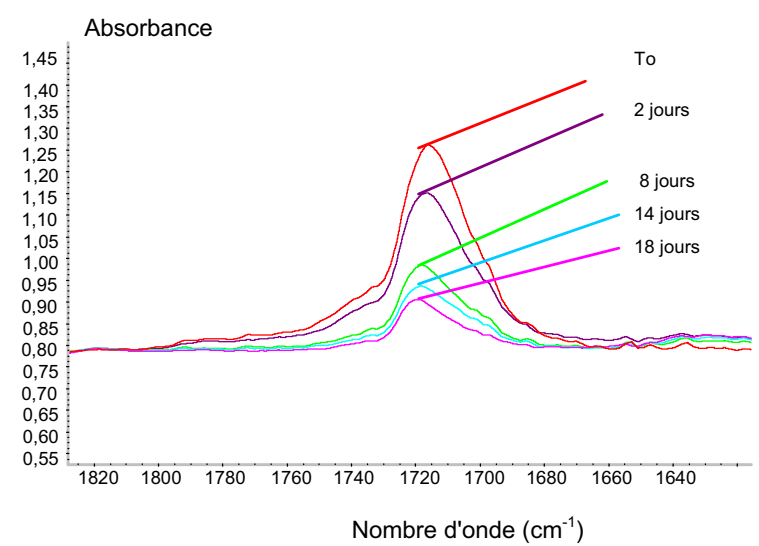

(b)

Fig. 2. Évolution des spectres IRTF-Transmission de films de PEHD préoxydés puis incubés : (a) dans un lixiviat d'ISDND biotique en conditions aérobies, (b) dans un lixiviat d'ISDND abiotique en conditions aérobies, zoom dans la région des produits d'oxydation de types carbonyles $(\mathrm{CO})$.

Fig. 2. FTIR spectra in transmission mode of PE films pre oxidized and incubated in: (a) biotic Municipal Solid Waste (MSW) landfill leachate under aerobic conditions, (b) abiotic MSW landfill leachate under aerobic conditions, zoom in the carbonyl (oxidation products) region.

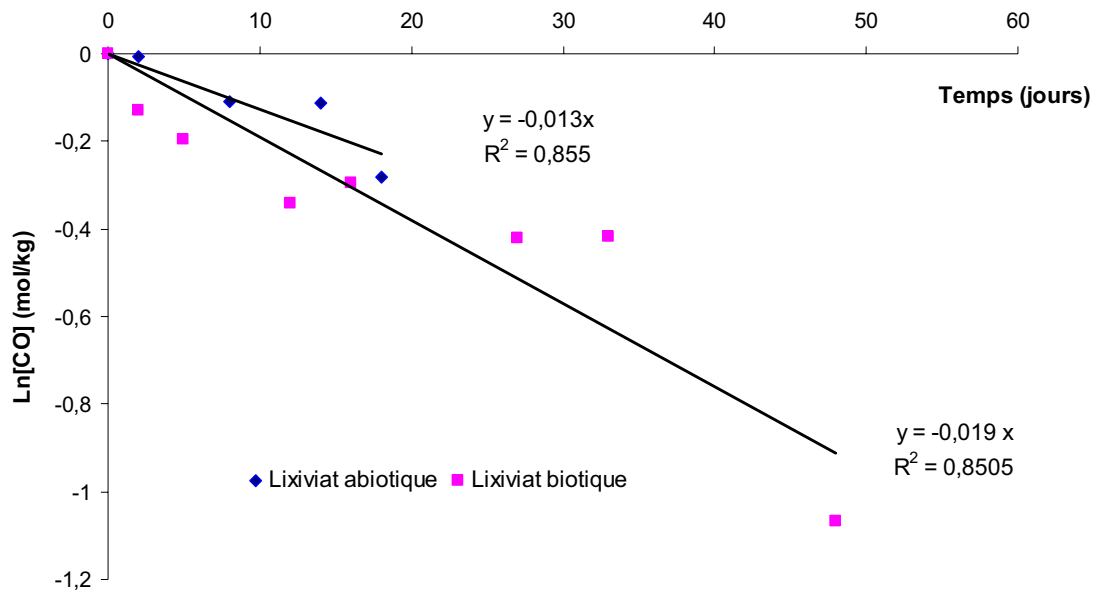

Fig. 3. Cinétique d'extraction des CO d'échantillons de PEHD préoxydés et incubés dans du lixiviat biotique ou abiotique, en conditions aérobies.

Fig. 3. Kinetic of carbonyl extraction from pre oxidized HDPE samples incubated in biotic and abiotic MSW landfill leachate, under aerobic conditions.

\subsection{Quantification des produits carbonylés extraits}

D'après Albertsson et al. [5], les acides carboxyliques correspondent aux produits de dégradation majoritairement formés au cours de l'oxydation du polyéthylène. La connaissance des mécanismes mis en jeu au cours du vieillissement des PE dans les ISDND nécessite donc de quantifier les acides carboxyliques extraits.

Dans cet objectif, nous avons utilisé la spectrophotométrie infra-rouge après déri- vatisation par traitement gazeux à l'ammoniac. Les échantillons de PEHD préoxydés puis incubés dans du lixiviat biotique à différentes échéances, ont été exposés dans du $\mathrm{NH}_{3}$ gazeux, à température ambiante durant $72 \mathrm{~h}$.

La réaction entre les acides carboxyliques $(\mathrm{RCOOH})$ et l'ammoniac $\left(\mathrm{NH}_{3}\right)$ est donné par l'équation (3) suivante :

$$
\mathrm{RCOOH}+\mathrm{NH} 3 \rightarrow \mathrm{RCO}_{2}^{-}, \mathrm{NH}_{4}^{+} .
$$




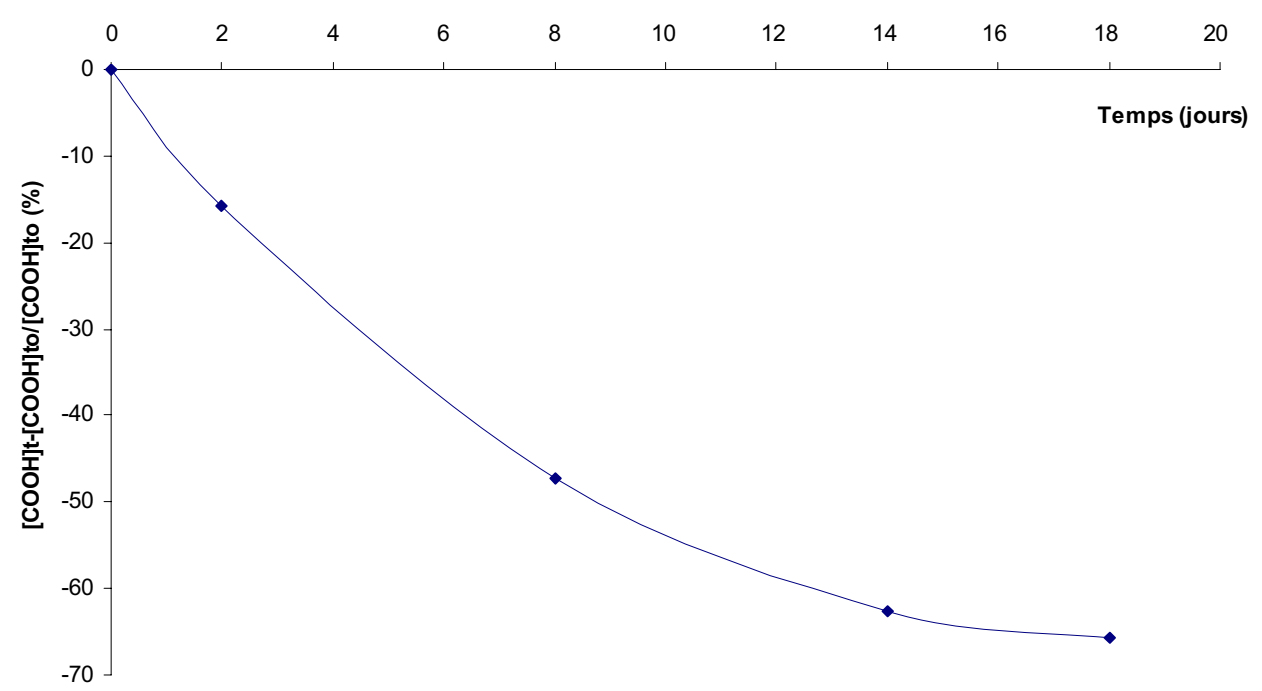

Fig. 4. Évolution de la concentration en acides carboxyliques de films de PEHD préoxydés et incubés dans du lixiviat.

Fig. 4. Variation of carboxylic acids concentration of HDPE films pre oxidized and incubated in landfill leachate.

Les carboxylates formés $\left(\mathrm{RCOO}^{-}\right)$sont aisément identifiables par leur bande d'absorption infra-rouge à $1576 \mathrm{~cm}^{-1}$; l'apparition de cette bande s'accompagne d'une diminution du massif situé entre 1800 et $1650 \mathrm{~cm}^{-1}$, caractéristique des composés carbonylés. Le dosage des acides carboxyliques est alors possible en soustrayant les spectres avant et après dérivatisation.

La perte relative en acides carboxyliques en fonction du temps d'incubation dans le lixiviat est représentée sur la figure 4 .

Par application de la loi de Beer-Lambert à $1715 \mathrm{~cm}^{-1}$ avec un coefficient d'absorption molaire $\varepsilon=6801 . \mathrm{mol}^{-1} \cdot \mathrm{cm}^{-1}$ [20], on détermine la concentration en acides carboxyliques à l'issue de l'oxydation. En considérant que la formation de deux moles de $\mathrm{COOH}$ est associée à une coupure de chaîne (n), l'utilisation des équations de SAITO [23] (Éqs. (4) et (5)) permet alors de déterminer la masse molaire moyenne en poids $\left(M_{W}\right) \mathrm{du}$ PEHD après pré-oxydation.

$$
\begin{aligned}
M_{n} & =\left(\frac{n(t)}{2}+\frac{1}{M_{n 0}}\right)^{-1} \\
M_{W} & =P I\left(\frac{n(t)}{2}+\frac{1}{M_{n 0}}\right)^{-1} .
\end{aligned}
$$

Après pré-oxydation, le polyéthylène présente donc une masse molaire moyenne en poids de $22,68 \mathrm{~kg} \cdot \mathrm{mol}^{-1}$. D'après la littérature, seules les courtes chaînes de PE dont la masse molaire moyenne en poids est inférieure à $20-25 \mathrm{~kg} \cdot \mathrm{mol}^{-1}$ sont assimilables par les microorganismes $[6,8,11]$. Il semblerait donc ici que les espèces carbonylées extraites dans le lixiviat soient susceptibles d'être ingérées par les microorganismes afin de favoriser leur développement.

\subsection{Croissance d'un biofilm à la surface du polyethylène}

Les surfaces des films de PEHD préoxydés et non oxydés ont été observées par Microscopie Électronique à Balayage (MEB) (Fig. 5). Les clichés MEB des films de PEHD préoxydés et incubés dans le lixiviat durant 7 mois (c), mettent en évidence la présence d'importants agrégats hétérogènes qui correspondent très certainement à des amas de microorganismes qui colonisent la surface du polymère sous forme de biofilm. Des résultats similaires ont été obtenus pour des incubations de films de PEHD et PEBD avec des souches pures non spécifiques du lixiviat $[9,11]$ ou des milieux complexes tels que des composts ou des sols [19]. La formation d'un biofilm n'implique pas nécessairement la biodégradation du matériau [24]. Néanmoins, dans la majorité des cas, le métabolisme des microorganismes présents dans un biofilm est plus important que lorsqu'ils sont sous forme planctonique [25]. 

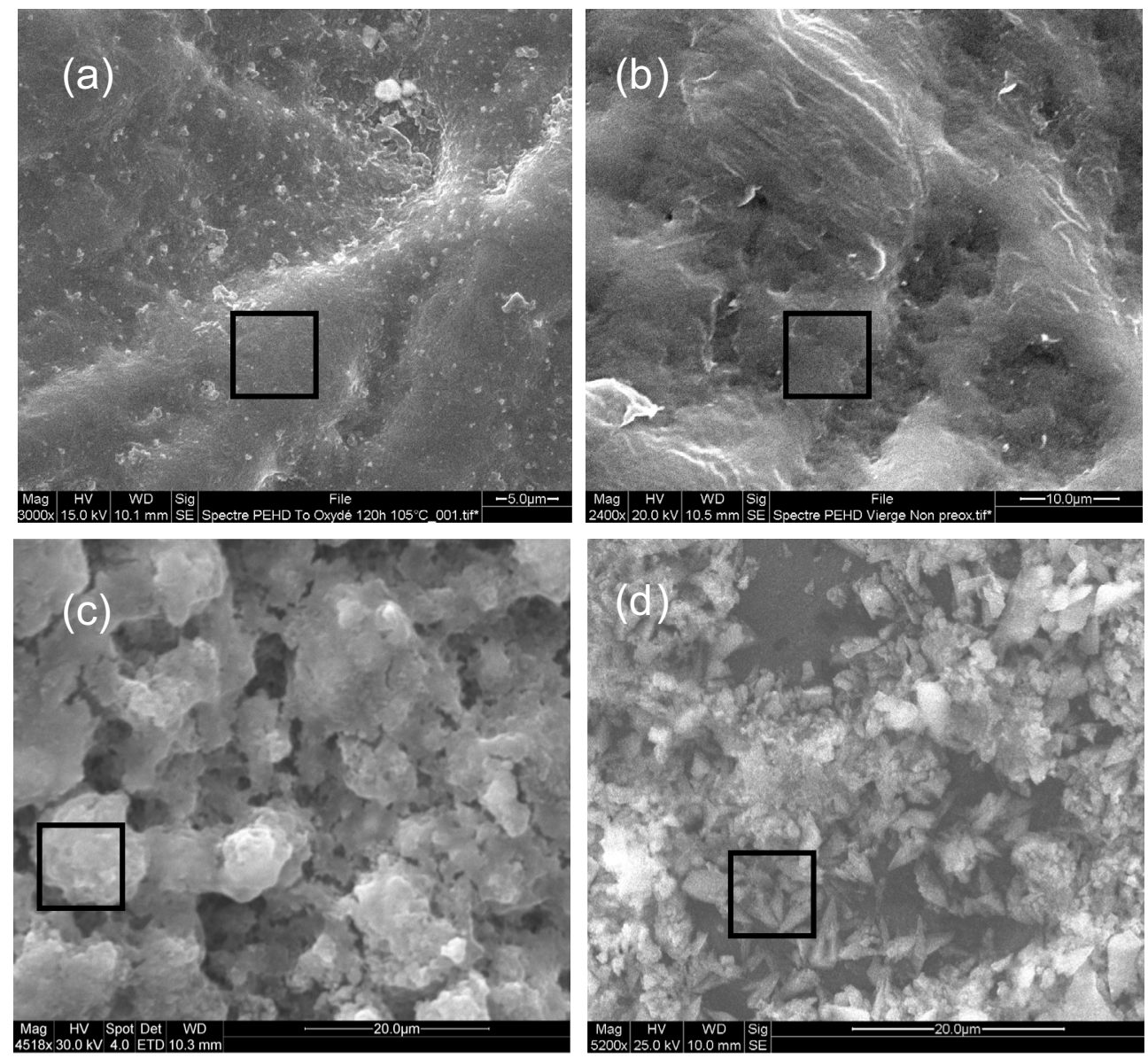

Fig. 5. Clichés MEB de la surface de films de PEHD (a) préoxydé avant incubation; (b) neuf non préoxydé avant incubation; (c) préoxydé et incubé 7 mois dans du lixiviat aérobie ; (d) non préoxydé et incubé 7 mois dans du lixiviat aérobie.

Fig. 5. SEM images of HDPE films surface: (a) pre oxidized before incubation, (b) unoxidized before incubation, (c) preoxidized and incubated 7 months in the aerobic leachate; (d) unoxidized and incubated 7 months in the aerobic leachate.

Sur le cliché (d) de la figure 5, nous pouvons observer la présence de nombreux cristaux à la surface du PEHD non oxydé et incubé 7 mois dans du lixiviat. Par microanalyse $X$ (Tab. 1), nous constatons que ces cristaux sont majoritairement constitués de carbone, d'oxygène et de calcium. Ces résultats laissent donc penser qu'à la surface du PEHD non préoxydé et incubé dans du lixiviat, se trouvent des précipités de carbonates de calcium $\left(\mathrm{CaCO}_{3}\right)$ provenant du lixiviat. En effet, durant le métabolisme anaérobie des microorganismes, du dioxyde de carbone est produit. Selon le $\mathrm{pH}$ du lixiviat, un équilibre entre la quantité de dioxyde de carbone et de carbonates s'établit [26].

Les résultats de la microanalyse $X$ du biofilm présent à la surface du PEHD préoxydé
(Tab. 1) montrent quant à eux, que le biofilm est constitué de substances organiques et minérales. Ces résultats sont en accord avec ceux présents dans la littérature [27], qui mettent en évidence cette caractéristique pour l'ensemble des biofilms étudiés, alors même que certains minéraux ne sont présents qu'à l'état de trace dans le milieu d'incubation.

L'existence d'un biofilm à la surface du PEHD préoxydé a également été prouvée par la technique de spectrophotométrie IRTF-ATR (Fig. 6). L'analyse de la surface du film vieilli 48 jours met en évidence la présence de bandes d'absorption entre 3000$3600 \mathrm{~cm}^{-1}$ et $1653 \mathrm{~cm}^{-1}$ qui correspondent au matériel protéique ainsi qu'une bande dans la région $1200-900 \mathrm{~cm}^{-1}$ attribuable aux 
Tableau 1. Comparaison de microanalyses $X$ à la surface de différents échantillons de PEHD.

Table 1. X-ray microanalysis of different HDPE samples surface.

\begin{tabular}{ccccc}
\hline Échantillons & $\begin{array}{c}\text { ECH (a) : } \\
\text { Eléments } \\
\text { surface du PEHD } \\
\text { préoxydé }\end{array}$ & $\begin{array}{c}\text { ECH (b) : } \\
\text { surface du PEHD } \\
\text { non préoxydé }\end{array}$ & $\begin{array}{c}\text { ECH (c) : } \\
\text { biofilm à la surface } \\
\text { du PEHD préoxydé et } \\
\text { incubé dans du lixiviat }\end{array}$ & $\begin{array}{c}\text { ECH (d) : } \\
\text { cristaux à la surface } \\
\text { du PEHD non préoxydé } \\
\text { et incubé dans du lixiviat }\end{array}$ \\
\hline $\mathrm{C}$ & 87,15 & 88,71 & 21,65 & 32,76 \\
$\mathrm{O}$ & - & - & 37,47 & 42,54 \\
$\mathrm{Na}$ & - & - & 2,58 & - \\
$\mathrm{Mg}$ & - & - & 8,90 & 0,68 \\
$\mathrm{Si}$ & - & 11,29 & 14,77 & 0,61 \\
$\mathrm{Au}$ & 12,85 & - & 10,11 & 6,70 \\
$\mathrm{Cl}$ & - & - & 0,80 & - \\
$\mathrm{K}$ & - & - & 1,61 & - \\
$\mathrm{Ca}$ & - & 2,11 & 16,71 \\
\hline
\end{tabular}

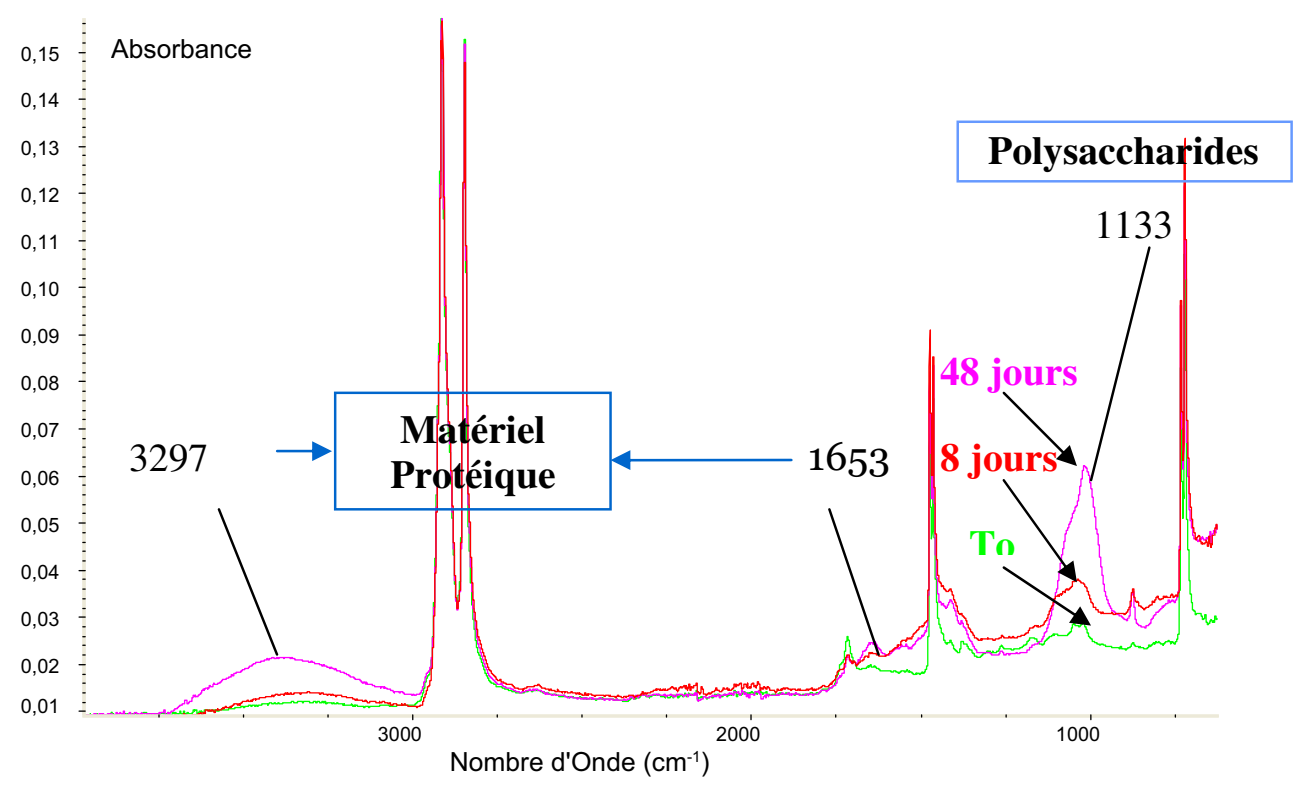

Fig. 6. Évolution des spectres IRTF-ATR de films de PEHD préoxydés et incubés dans du lixiviat biotique d'ISDND en conditions aérobies.

Fig. 6. FTIR spectra in ATR mode of pre oxidized HDPE films incubated in biotic MSW landfill leachate under aerobic conditions.

polysaccharides, constituants cellulaires des microorganismes. L'évolution des spectres de la figure 6 après différents temps de contact des échantillons met en évidence une augmentation des bandes caractéristiques des microorganismes par un épaississement du biofilm. En revanche, pour les films de PEHD non oxydés également incubés dans du lixiviat en conditions aérobies, les spectres IRTF-ATR (Fig. 7) ne comportent pas les bandes caractéristiques du matériel protéique situées entre 3000 et $3600 \mathrm{~cm}^{-1}$ et $1653 \mathrm{~cm}^{-1}$. Cependant, on note la présence $\mathrm{d}^{\prime}$ une très faible absorption entre $1200-900 \mathrm{~cm}^{-1}$ sur le spectre du PEHD d'ori- gine, qui ne semble pas évoluer après incubation. Ces bandes sont sans doute dues à la présence de bactéries existantes sur le film de polymère d'origine qui n'a pas été stérilisé avant les essais de vieillissement. Deux hypothèses peuvent expliquer les tendances observées :

(i) La croissance bactérienne à la surface du PEHD préoxydé est accélérée par la présence de composés carbonylés bio assimilables, créés lors du mécanisme d'oxydation.

(ii) La faible adhésion des microorganismes à la surface du PEHD non oxydé, et 


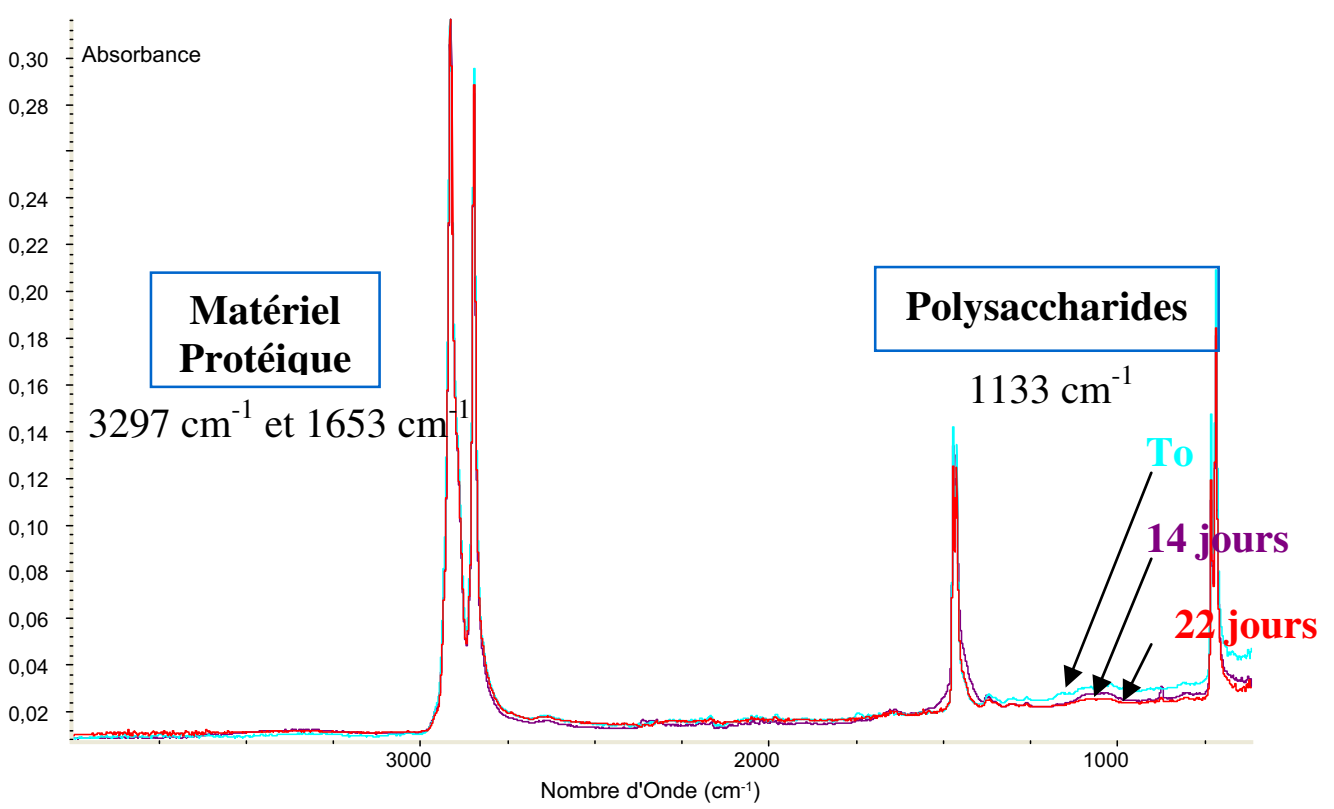

Fig. 7. Évolution des spectres IRTF-ATR de films de PEHD non préoxydés et incubés dans du lixiviat biotique d'ISDND en conditions aérobies.

Fig. 7. FTIR spectra in ATR mode of unoxidized HDPE films incubated in biotic MSW landfill leachate under aerobic conditions.

par conséquent hydrophobe, est certainement liée au caractère hydrophile de la surface des microorganismes présents dans le lixiviat (Hadad, 2005).

\section{Conclusion}

La technique d'analyse spectrophotométrique d'infra-rouge à Transformée de Fourier (IRTF) en mode transmission a permis de mettre en évidence une extraction des composés carbonylés de faibles masses molaires formés lors de l'oxydation des chaînes du PEHD dans un lixiviat biotique et abiotique. Dans les deux milieux, les vitesses d'extraction sont de l'ordre de 1,5 $\times$ $10^{-2}$ jours $^{-1}$.

Les acides carboxyliques sont les composés majoritairement formés lors de la dégradation oxydative des PEHD [5]. À l'aide de la méthode de dérivatisation par l'ammoniac, on montre une perte rapide de ces composés au cours de l'incubation du PEHD oxydé dans le lixiviat (65\% après 18 jours).

Les techniques d'IRTF en mode ATR et de MEB ont mis en évidence que la croissance bactérienne sous forme de biofilm est accélérée par la présence de composés carbony- lés de faibles tailles créés lors du mécanisme d'oxydation.

\section{Remerciements}

Cette étude fait partie du projet DURAGEOS dans le cadre du programme C2D2 du réseau RGCU du MEEDM, labellisé par les pôles de compétitivité ADVANCITY et AXELE.

\section{Références}

[1] R.K. Rowe, Geotechnique 5 (2005) 631-678

[2] G.R. Koerner, R.M. Koerner, Geotextiles and Geomembranes 24 (2006) 72-77

[3] H. Yoshida, R.K. Rowe, Consideration of landfill liner temperature, in T.H. Christensen et al. (Eds.), Proceedings of the Ninth International Waste Management and Landfill Symposium, CISA, Italy (CDROM), 2003

[4] R.M. Koerner, G.R. Koerner, A.W. Eith, C.P. Ballod, Geomembrane temperature monitoring at dry and wet landfills, in Global Waste Management Symposium, Colorado, USA (CD-ROM), 2008

[5] A.C. Albertsson, C. Barenstedt, S. Karlsson, T. Lindberg, Polymer 36 (1995) 3075-3083

[6] A.C. Albertsson, B. Erlandsson, M. Hakkarainen, S. Karlsson, J. Environ. Polym. Degrad. 6 (1998) 4 
[7] M. Weiland, A. Daro David, Polym. Degrad. Stabil. 48 (1995) 275-289

[8] K. Yamada-Onodera, H. Mukumoto, Y. Katsuyaya, A. Saiganji, Y. Tani, Polym. Degrad. Stabil. 72 (2001) 323-327

[9] S. Bonhomme, A. Cuer, A.-M. Delort, J. Lemaire, M. Sancelme, G. Scott, Polym. Degrad. Stabil. 81 (2003) 441-452

[10] I. Jakubowicz, Polym. Degrad. Stabil. 80 (2003) 39-43

[11] M. Koutny, M. Sancelme, C. Dabin, C. Pichon, Polym. Degrad. Stabil. 91 (2006) 14951503

[12] E. Chiellini, A. Corti, S. D'Antone, Polym. Degrad. Stabil. 92 (2007) 1378-1383

[13] A.C. Albertsson, S.O. Andersson, S. Karlsson, Polym. Degrad. Stabil. 18 (1987) 73-87

[14] J. Pospisil, Polym. Degrad. Stabil. 40 (1993) 217-232

[15] H. Zweifel, Effect of stabilization of polypropylene during processing and its influence on long term behaviour under thermal stress, Polymer Durability, Degradation, Stabilization and Lifetime prediction, American Chemical Society, 1996

[16] E. Richaud, Durabilité des géotextiles en polypropylène, Thèse de doctorat de l'École Nationale Supérieure des Arts et Métiers, France, 2006

[17] Y. Kamiya, E. Niki, Aspects of degradation and stabilization of polymers, chap. Oxidative Degradation, Ed. H.H.G. Jellinek, 1978, pp. 79-147
[18] D. Hadad, S. Geresh, A. Sivan, J. Appl. Microbiol. 98 (2005) 1093-1100

[19] T.F.M. Ojeda, E. Dalmolin, M.M.C. Forte, R.J.S. Jacques, F.M. Bento, F.A.O. Camargo, Polym. Degrad. Stabil. 94 (2009) 965-970

[20] F.M. Rugg, J.J. Smith, R.C. Bacon, J. Polym. Sci. 13 (1954) 535-547

[21] K. Maquelin, C. Kirschner, L.-P. ChooSmith, N. Van den Braak, H.Ph. Endtz, D. Naumann, G.J. Puppels, J. Microbiol. Meth. 51 (2002) 255-271

[22] P.D. Calvert, N.C. Billingham, J. Appl. Polym. Sci. 24 (1979) 357-370

[23] O. Saito, The radiation chemistry of macromolecules, Ed. M. Dole, Academic Press, NY, 1972

[24] J.P. Eubeler, M. Bernhard, T.P. Knepper, Environmental biodegradation of synthetic polymers, Part. II: Biodegradation of different polymer groups, Trends Anal. Chem., 2009

[25] D. Kirchman, R. Mitchell, Appl. Environ. Microbiol. 43 (1982) 200-209

[26] P. Kjeldsen, M.A. Barlaz, A.P. Rooker, Environ. Sci. Technol. 32 (2002) 297-336

[27] F. Fritz-feugeas, A. Cornet, B. Tribollet, Biodétérioration des matériaux : Action des microorganismes, de l'échelle nanométrique à l'échelle macroscopique, Édition Ellipses, 2008 